\title{
Plan de desarrollo de la pesquería en el Perú
}

Fernando Kleeberg

Ingeniero pesquero por la Universidad Nacional Agraria La Molina. Máster en ingeniería de alimentos por la Universidad de Campinas, Brasil. Es miembro del Institute of Food Technologists de Estados Unidos y del International Institute of Refrigeration de Francia.

Ejerce la docencia en la Universidad de Lima

El sector pesquero se está preparando para afrontar los retos del tercer milenio, elaborando planes estratégicos que permitirán desarrollar una pesquería sostenida en el tiempo. Estos planes están dirigidos a maximizar el aprovechamiento de las materias primas así como a la preservación de éstas, alentar procesos productivos eficientes e incentivar la capacitación y preparación de los trabajadores. Uno de los puntos resaltantes es fomentar la elaboración de productos destinados al consumo humano directo, con el fin de incrementar el valor agregado y llegar a ser lideres en exportaciones pesqueras, asi como promover el incremento de la acuicultura, ya que el país cuenta con más de 850 especies ícticas en aguas dulces. 


\section{Introducción}

El Ministerio de Pesquería y los entes representativos de los industriales del sector, se han reunido y planteado los aspectos más importantes que permitirán el desarrollo de una pesquería sostenida en el tiempo. El presente artículo ha sido elaborado sobre la base de dos conferencias realizadas en la Universidad de Lima: "Plan estratégico 1999-2004", ofrecida por Juan Alberto Arrús, viceministro de Pesquería, y "Perspectivas del desarrollo de la acuicultura en el Perú", de Enrique Vinatea, investigador y experto en acuicultura.

Para el desarrollo del plan estratégico ha sido muy importante la capacidad negociadora de Ludwig Meier, ex ministro de Pesquería, quien posee una clara visión de lo que debe ser este sector. En una de sus participaciones, como ministro, manifestó que el desenvolvimiento de las actividades pesqueras en forma eficiente implica, necesariamente, buscar el máximo aprovechamiento de las materias primas, promover procesos productivos de alta calidad, así como crear una flota idónea para la extracción y contar con cuadros técnicos y profesionales capacitados. Todo ello complementado con la preservación, que implica respeto por los recursos y el cuidado escrupulosos del medio ambiente.

\section{Importancia del plan estratégico}

La importancia de contar con objetivos de mediano y largo plazos está asociada al concepto de recursos naturales renovables.

En diversas partes del mundo se depredaron diferentes recursos porque se buscó obtener el máximo beneficio en el corto plazo o porque no se contó con herramientas adecuadas para evitarlo.

Esta depredación se intentó evitar mediante vedas, cuotas y restricciones, que no siempre funcionaron. El principal motivo ha sido que el regulador no era consecuente, su política cambiaba en cada administración y no se contaba con un planeamiento estratégico adecuado.

Para el desarrollo sostenido de un sector, los objetivos y planes de acción deben trascender a la administración de turno. Por tanto, es necesario implementar un plan estratégico que 
permita alcanzar los objetivos establecidos, ejecutando y adecuando los planes de acción a las contingencias.

\section{Sector pesca: plan estratégico}

La validez del plan estratégico del sector pesca se sustentará en tres parámetros fundamentales:

- Ser el resultado de un trabajo conjunto entre los sectores público y privado.

- Su difusión y explicación a todos los agentes del sector.

- El compromiso de cumplirlo que adquieran los agentes del sector.

El plan estratégico se origina con la misión, que es la razón de ser del sector y el código de conducta a seguir por todos sus agentes.

La visión es una descripción de cómo se encontraría el sector al término del horizonte de planeamiento (año 2004).

El análisis Foda (fortalezas, oportunidades, debilidades y amenazas) es el diagnóstico de las fases y recursos del sector.

La matriz de acción es el paso previo al diagnóstico, y tiene un carácter dinámico. Aquí se determina qué se debe mantener, evitar o mejorar en la situación actual y, por otro lado, qué se deberá lograr o prevenir.

Los objetivos y sus respectivos lineamientos deberán ser el eje de los planes de acción durante el período 1999-2004.

\section{Misión}

Desarrollar las actividades pesqueras en forma competitiva, eficiente y sostenible en el tiempo, preservando los recursos hidrobiológicos y protegiendo a la vez su medio ambiente.

\section{- De forma competitiva y eficiente}

- Máximo aprovechamiento de la materia prima.

- Procesos productivos con alta calidad.

- Flota idónea para la extracción diversificada.

- Cuadros técnicos y profesionales capacitados.

\section{- Preservación}

- Antónimo de depredación (respeto a los recursos).

- Crecimiento de los recursos (mar y aguas continentales).

- Cuidado del medio ambiente. 


\section{Visión del 2004}

El Perú es un país líder en exportaciones pesqueras, lo que se sustenta en el incremento del valor agregado de las mismas y en el continuo desarrollo de productos destinados al consumo humano directo. La acuicultura comienza a ser una actividad significativa en este sector industrial.

Las actividades pesqueras se realizan respetando los ecosistemas. Los recursos hidrobiológicos son más y mejor utilizados sin riesgo de disminuir su cantidad y calidad.

El Ministerio de Pesquería ha implementado una regulación efectiva y eficiente que promueve la investigación y el desarrollo de la actividad, en armonía con la sostenibilidad de los recursos.

Los agentes vinculados al sector se han comprometido con el desarrollo del factor humano, por lo que la capacidad y preparación de todos sus trabajadores se ha incrementado considerablemente; esto se refleja en mayor productividad y mejor calidad de vida.

El nivel de consumo de pescado en la población peruana se acrecienta en respuesta a la oferta de productos, la promoción de éstos y la mejora en las redes de distribución, generando así un mayor nivel de nutrición en la población.

Análisis de las fortalezas, oportunidades, debilidades y amenazas del sector pesquero en el Perú (Foda)

Para realizar el análisis Foda se deben considerar los seis factores principales que influyen de una manera significativa sobre la pesca en el país:

- Biomasa

- Extracción

- Procesamiento

- Acuicultura

- Comercialización

- Administración

Biomasa (recursos hidrobiológicos)

- Fortalezas

- Mar con alto nivel de fertilidad y de nutrientes.

- Diversidad de recursos.

- Pronta reposición de los stocks de anchoveta (autorrenovabilidad). 


\section{- Debilidades}

- Tres recursos plenamente explotados: anchoveta, sardina y merluza (aprox. 94\% de captura).

- Limitada investigación científica sobre fenómenos oceanográficos recurrentes y sus efectos.

- Escasa investigación sobre otras especies.

- Aparente poca disponibilidad de recursos inexplotados.

- Oportunidades

- Aprovechar eficientemente los recursos existentes.

- Desarrollar las especies subexplotadas e inexplotadas.

- Aprovechar las especies de oportunidad (los fenómenos oceanográficos).

- Amenazas

- Exceso de capacidad de bodega destinada a la pesca de anchoveta y sardina que conduciría a sobreexplotación.

- Fenómenos oceanográficos que conducen a migración de especies y/o afectan ciclos reproductivos.

- Contaminación afecta los ecosistemas costeros y la acuicultura.

\section{Extracción}

- Fortaleza

- Condiciones de mar favorables.

- Caladeros cercanos a la costa.

- Capacidad y flexibilidad de la flota (convertibilidad hacia otras pesquerías).

- Infraestructura de apoyo ligada a la flota cerquera.

- Experiencia en pesca con flota cerquera (país líder mundial).

- Infraestructura de desembarque funcional y de apoyo a la flota pesquera artesanal.

\section{- $\quad$ Debilidades}

- Insuficientes embarcaciones para pesquerías distintas a la anchoveta, sardina y merluza.

- Insuficiencia y falta de preparación de cuadros (mandos y personal embarcado) en flota industrial. Edad promedio cercana a jubilación. 
- Falta de responsabilidad y respeto al recurso.

- Sobredimensionamiento de la flota, ineficiencia de parte de ella y concentrada en dos recursos.

- Tendencia a la pesca costera, limitando el radio de acción de la mayor parte de la flota cerquera (un día).

- Insuficiente infraestructura adecuada para desembarque de pesca de consumo humano directo.

- Deficiencias de control.

- Oportunidades

- Desarrollo de pesquerías de media agua, profundidad y altura.

- Conversión y diversificación de la flota cerquera existente.

- Modernización de la flota artesanal.

- Amenazas

- Agotamiento de los recursos.

- Ingreso ilegal de embarcaciones extranjeras en aguas jurisdiccionales.

- Acceso ilegal de embarcaciones hacia pesquerías plenamente explotadas.

- Reducido desarrollo de pesca artesanal por falta de actitud empresarial motivada por el asistencialismo estatal.

- Restricciones internacionales.

\section{Procesamiento}

- Fortalezas

- Capacidad instalada con tendencia a la modernización.

- Versatilidad: posibilidad de aumento de líneas de producción.

- Sistemas de aseguramiento de la calidad, principalmente en congelado y conservas.

- Empresas con plantas distribuidas a lo largo del litoral.

- Debilidades

- Falta de condiciones para motivar al empresario a la diversificación hacia consumo humano directo.

- Se realiza gestión de corto plazo debido a alta rentabili- 
dad en harina y aceite de pescado.

- Insuficiencia de sistemas de preservación de la materia prima.

- Insuficiente infraestructura adecuada para procesamiento de pesca artesanal.

- Oportunidades

- Diversificación para consumo humano directo

- Opción de incrementar beneficios vía mejoras en eficiencia de procesos.

- Disposición de recursos para la diversificación de la industria pesquera.

- Demanda externa creciente de productos pesqueros procesados de alta calidad.

- Amenazas

- Actividad económica concentrada en un solo producto.

- Casos de incumplimiento de estándares de calidad en harina (porque no existe rechazo, sólo penalización de precio), afectan a las exportaciones.

Acuicultura

- Fortalezas

- Litoral y zona continental amplios.

- Variedad de microclimas.

- Producción puede planificarse (menor aleatoriedad).

- Experiencia en cultivos de langostino, concha de abanico, trucha y camarón de Malasia.

- Aceptación de productos en el mercado.

- Centros experimentales de adaptación de tecnologías de cultivo de recursos hidrobiológicos en zonas marinas y continentales.

- Debilidades

- Falta de coordinación de políticas intersectoriales.

- Legislación actual requiere modificación que promueva iniciativas privadas de desarrollo.

- Acondicionamiento de la mayoría de zonas requiere de más inversión que en otros países.

- Limitaciones en infraestructura de transporte. 
- Incipiente uso de tecnología para la crianza de peces.

- Oportunidades

- Alto potencial en la selva y a lo largo del litoral por diversidad de especies.

- Aprovechar la iniciativa privada existente.

- Múltiple desarrollo de tecnologías transferibles.

- Demanda global creciente a consecuencia de la necesidad de alimentos con alto valor proteico.

- Amenazas

- Contaminación (minera, agraria y urbana).

- Especulación pública y privada en otorgamiento de concesiones eleva precios desanimando la inversión.

- Poco conocimiento en el control y tratamiento de enfermedades.

- Otorgamiento de concesiones en bancos naturales.

\section{Comercialización}

- Fortalezas

Comercialización externa.

- País pesquero reconocido mundialmente.

- Alta demanda de harina y aceite de pescado por utilización en acuicultura.

- Buen posicionamiento de productos de consumo humano.

Comercialización interna

- Variedad de productos de consumo masivo.

- Demanda bastante sensible al precio.

- Relativa disponibilidad de flota de transporte para productos hidrobiológicos del segmento pesquero artesanal.

- Debilidades

Comercialización externa

- Incumplimiento de algunos productores genera mala imagen.

- Especies actualmente explotadas tienen poco valor de mercado.

- Altos costos afectan competitividad de productos de consumo humano. 
- Falta de promoción del valor proteico de la harina de pescado.

Comercialización interna

- Insuficiente infraestructura adecuada, principalmente carreteras y sistemas de frío.

- Insuficientes centros de distribución mayoristas.

- Puntos de venta minoristas poco atractivos.

- Deficiente sistema de información de precios.

- Altos costos afectan comercialización de productos de consumo humano.

- Oportunidades

Comercialización externa

- Labor promotora de Prompex para llegar a nuevos mercados.

- Demanda creciente por la existencia de mercados insatisfechos.

- Integración del país a bloques económicos internacionales.

- Potencial de nuevos recursos y productos.

Comercialización interna

- Demanda de productos de consumo humano directo insatisfecha y en crecimiento.

- Amenazas

Comercialización externa

- Aparición de sustitutos para la harina de pescado.

- Incremento de barreras paraarancelarias (estándares, ecología, sanidad, biodiversidad, etc.).

- Desmantelamiento del sistema generalizado de preferencias arancelarias (UE y EE UU).

- Rechazo por incumplimiento de estándares de calidad, sanidad, higiene.

Comercialización interna

- Desastres naturales que afecten el acceso al mercado.

- Incumplimiento de la calidad de productos.

\section{Administración}

- Fortalezas

- Restablecimiento del principio de autoridad.

- Participación activa del sector privado. 
- Ordenamiento que facilita la gestión pesquera.

- Capacidad de reacción ante coyunturas.

- Organismos descentralizados con prestigio internacional.

- Apoyo en obras de infraestructura.

- Debilidades

- Falta de continuidad en las políticas.

- Falta de coordinación interinstitucional y gremial.

- Carencia de cuadros especializados en la administración y en los organismos descentralizados.

- Reducida autonomía en la gestión de las instituciones por la poca flexibilidad.

- Falta mejorar los sistemas de acceso a las pesquerías.

- Falta mejorar la red de control y vigilancia.

- Oportunidades

- Potencial de desarrollo de organismos descentralizados.

- Captación de recursos para ejecución de obras de infraestructura y desarrollo de proyectos.

- Coyuntura permite modernizar el sistema de administración.

- Integración de las redes de información.

- Amenazas

- Tentación de exceso de controles.

- Discontinuidad de las políticas por cambio de administración.

- Migración de profesionales calificados por búsqueda de mejores condiciones económicas.

Matriz de acción

- ¿Qué es necesario mejorar?

- Las investigaciones que tienen por fin preservar la biomasa y realizar acciones para sostenerla.

- Los sistemas de acceso a las pesquerías.

- La calidad de la extracción buscando preservar las especies.

- El procesamiento de los productos marinos.

- El desarrollo de la acuicultura.

- El desarrollo de la pesca artesanal. 
- Los sistemas de proyección y pronósticos de las fluctuaciones del ambiente marino.

- La comercialización de productos.

- La capacitación de los trabajadores.

- Los organismos descentralizados.

- El marco regulatorio interinstitucional.

- ¿Qué se debe prevenir / evitar?

- La sobreexplotación de las especies, el deterioro del ecosistema y la contaminación del medio ambiente.

- Los bajos índices de conversión y calidad.

- El uso de artes y aparejos de pesca no adecuados.

- El uso de técnicas y condiciones inadecuadas para la transformación de productos de consumo humano.

- La continua migración de profesionales calificados a otros sectores en busca de mejores condiciones.

- La contaminación de bahías, caletas, lagos y lagunas aptas para la acuicultura.

- El ingreso ilegal de embarcaciones en aguas jurisdiccionales y los acuerdos internacionales que limiten la extracción de determinados recursos.

- ¿ ¿ué se requiere mantener?

- El liderazgo como país pesquero en volumen de captura y producción.

- La riqueza ictiológica del mar peruano.

- El volumen de anchoveta, sardina y merluza.

- Mantener a la harina de pescado especial como uno de los principales productos.

- ¿Qué se busca lograr?

- Liderazgo como país pesquero.

- La sostenibilidad de los recursos hidrobiológicos a partir de su aprovechamiento óptimo.

- Que todas las personas naturales y jurídicas cumplan con el manual de buenas prácticas, con normas de sanidad y calidad.

- Contar con una flota pesquera moderna, eficiente, multipropósito, con sistemas de preservación principalmente para los productos de consumo directo para así garantizar la calidad de las materias primas. 
- Incrementar las exportaciones de productos de consumo humano directo y de harinas especiales, mejorando la estructura actual de las exportaciones pesqueras.

- Contar con productores permanentes en acuicultura: langostinos, concha de abanico, camarón de Malasia, truchas, algas, etc.

- Usar el alto potencial en zonas de ceja de selva y selva baja

- Tener una red eficiente de distribución y puntos de venta en todo el país.

- Contar con trabajadores conscientes del manejo de recursos.

- Formar cuadros técnicos y profesionales para todos los niveles.

- Ampliar la oferta exportable. Incorporar anchoveta para consumo humano.

- Comprender y aplicar el Código de Conducta de Pesca Responsable.

- ¿Cuáles son los ideales?

- Lograr que la mayor parte de la materia prima tenga calidad suficiente para consumo humano.

- Que el valor de las exportaciones de conservas y congelados sea equivalente o mayor al de las exportaciones de harina y aceite de pescado.

- Sortear eventos de fuerte variabilidad climática, sin que la industria sea afectada.

- Lograr aprovechar totalmente los recursos pesqueros.

- Incrementar el consumo per cápita de pescados y mariscos.

- Incrementar la cooperación entre el sector público y el privado.

- Contar con alto grado de capacitación en todos los niveles de los sectores público y privado.

\section{Objetivos y lineamientos}

- Objetivos

- Preservar la biomasa y los ecosistemas.

- Promover el uso diversificado de los recursos hidrobiológicos y la generación de mayor valor. 
- Desarrollar la acuicultura, nuevas pesquerías y pesca artesanal.

- Mejorar la gestión del ministerio y de los organismos descentralizados.

- Objetivo 1: Preservación de la biomasa y los ecosistemas

- Fomentar la pesca responsable en aguas marinas y continentales, mediante sistemas y planes de ordenamiento que garanticen la sostenibilidad de los recursos.

- Promover e incentivar la investigación científica, tecnológica y capacitación pesquera a través de organismos especializados, universidades e iniciativa privada, dando prioridad a los recursos declarados plenamente explotados.

- Fomentar la preservación de la diversidad biológica y de la calidad de su ambiente, cautelando el cumplimiento de las medidas que reduzcan o eviten su deterioro.

- Objetivo 2: Propiciar el uso diversificado de los recursos hidrobiológicos y la generación de mayor valor

- En acciones concertadas entre el Estado y el sector privado, propender a la utilización integral y racional de los recursos hidrobiológicos, orientándolos al desarrollo de productos con mayor valor agregado.

- Orientar la operatividad de una moderna flota pesquera que cuente con artes y aparejos de pesca adecuados y sistemas de preservación, regulando y controlando el cumplimiento de normas de higiene y sistemas de preservación a bordo.

- Racionalizar la flota dedicada a la extracción de recursos pelágicos plenamente explotados a niveles compatibles con la captura permisible.

- Propiciar el mejoramiento de las condiciones higiénicosanitarias y la adopción de sistemas de aseguramiento de la calidad en las plantas de procesamiento y canales de distribución.

- Propiciar la cooperación técnica y económica nacional e internacional para facilitar el desarrollo del sector pesquero, intensificando los esfuerzos para la captación o incremento de recursos económicos hacia aquellas actividades de menor desarrollo y mayor potencial. 
Objetivo 3: Desarrollar la acuicultura, nuevas pesquerías y pesca artesanal

- Investigar para promover el desarrollo de nuevas pesquerías y actividades de acuicultura como base del aprovechamiento diversificado de las especies.

- Elevar la productividad de la pesca artesanal mediante la tecnificación y la capacitación de los recursos humanos.

- Desarrollar la infraestructura de la acuicultura a través de cooperación técnica internacional e inversión privada.

- Propiciar mecanismos promocionales para el desarrollo de la acuicultura y nuevas pesquerías.

Objetivo 4: Mejorar la gestión del ministerio y de los organismos descentralizados

- Brindar un mejor servicio a los usuarios simplificando y agilizando los procedimientos administrativos del Ministerio de Pesquería, organismos descentralizados y oficinas regionales.

- Fomentar el trabajo conjunto del sector público y el sector privado, incluyendo los temas internacionales.

- Establecer un régimen legal especial para la gestión institucional de los organismos descentralizados.

- Articular eficientemente los roles de los organismos descentralizados, optimizando su gestión y coordinación, promoviendo la participación del sector privado.

- Lograr mayor coordinación interinstitucional buscando un marco regulatorio integral.

Se considera adecuado incluir el gráfico $\mathrm{N}^{\circ} 1$ (véase anexo) de una industria pesquera limpia y competitiva que fue parte del trabajo de investigación "Industria de las principales especies de importancia económica del sector pesquero: anchoveta, sardina, jurel, caballa y merluza" realizada en la Universidad de Lima, donde con un gráfico causa-efecto se plantean los requisitos de una industria limpia y competitiva.

A continuación se presentan los aspectos más resaltantes de la conferencia de Enrique Vinatea, quien expuso sobre las perspectivas que tiene el Perú en el desarrollo de la acuicultura. 


\section{Perspectivas del desarrollo de la acuicultura en el Perú}

El Perú es uno de los primeros países pesqueros del mundo por el elevado volumen de recursos marítimos extraídos principalmente de su mar litoral y oceánico, que a su vez constituye uno de los más conspicuos por la gran productividad y riqueza de nutrientes que, al aflorar, fertilizan sus aguas superficiales.

Dispone también de recursos hidrobiológicos en fuentes continentales, así como animales posibles de asociarse al cultivo de peces. Existen 850 especies ícticas en las aguas dulces del Perú y se estima un aumento del $20 \%$ en el futuro.

Las familias de peces que sobresalen en la Amazonía son los carácidos (gamitana, paco, etc.), prochilóntidos (boquichicos), el gigante paiche (0,6-1,8 metros y 30-150 kilos) y los peces ornamentales.

En el lago Titicaca: suches, orestias, truchas y pejerrey argentino. En los ríos y lagunas de la sierra sobresalen los arriba mencionados. En la costa, los pocos ríos de caudal permanente producen peces y camarones.

A partir de los años treinta, la Dirección de Pesca y Caza del Ministerio de Agricultura promovió la introducción de varias especies en ríos y lagos del país.

\section{Selección de lugares y especies para la acuicultura}

Selección de lugares

- Lugares convenientes para las diversas clases de acuicultura

Consideraciones técnicas y no técnicas sobre la selección del lugar

- Factores socioeconómicos, políticos.

- Factores climáticos.

Factores ambientales principales

- Topografía.

- Suelo.

- Agua.

- Características del agua.

- Productividad: productividad acuática, métodos para medir la productividad.

- Incrustaciones. 
- Disponibilidad: larvas, alevinos y semillas en general.

- Clase y densidad de la vegetación.

Selección de especies

- Características deseables de los organismos acuícolas.

- Características biológicas de los organismos cultivados.

- Organismos comúnmente cultivados.

- Criterios para la selección de especies para el cultivo.

- Especies nativas respecto a las exóticas.

- Selección de variedades de especies cultivadas de características favorables.

En los gráficos $\mathrm{N}^{\circ} 2$ y $\mathrm{N}^{\circ} 3$ (véase anexo) se presentan: la clasificación de los recursos que pueden ser explotados en la acuicultura, así como los lugares y sistemas de cultivo adecuados.

\section{Zonas potenciales de la acuicultura en el Perú}

- Costa

- Litoral: maricultura con peces, moluscos, crustáceos, microalgas. Tumbes, Puerto Pizarro: apropiados para el cultivo de langostino (Penaeus vannamei, $P$. liforniensis, P. stylirostris).

- Posibilidad de ampliar la industria langostinera desde Piura hasta Tacna para introducir Penaeus paulensis, que soporta de $7-23^{\circ} \mathrm{C}$ resiste enfermedades, tiene crecimiento rápido. Esta especie es originaria del sur brasileño, especialmente de Santa Catarina, y de Argentina.

- $\quad$ Sierra

- Cuerpos de agua variados, lagunas altoandinas, como el lago Titicaca con población de trucha arco iris, pejerrey argentino; suche, ispis y peces menores.

- Entre las piscigranjas destacan El Ingenio y la piscifactoría Los Andes (Junín).

- Puno, Huánuco y Ancash destacan en trucha "arco iris".

- También pueden ser cultivados peces, crustáceos, camarones, cangrejos, cultivos integrados y rotativos, ranicultura.

- Selva alta y Selva baja

- Estudio previo para manejar potencial íctico-nativo.

- Piscicultura extensiva y de repoblamiento. 
- Aproximadamente 2.500 especies amazónicas: characiformes, siluriformes, perciformes, etc.

- El doctor Vinatea, en su publicación Piscicultura tropical de aguas continentales discute las características principales de paco, gamitana, tucunaré, acaraguasú, paiche, tilapias, carpas, etc.; que son especies interesantes de cultivar en esta zona.

- Selva baja. Iquitos y Pucallpa: especies nativas gamitana, paco, sábalo cola roja, paiche.

- Selva alta. Tarapoto, Moyobamba, Tingo María: paco, gamitana, boquichico, tilapia del Nilo, carpa común.

El Perú produce langostinos y truchas por un valor de 26,5 millones de dólares y tiene un amplio potencial para desarrollar la acuicultura; tanto como los grandes productores en el mundo: China, India, Japón, y otros países asiáticos que producen carpas, trucha, salmón y otros. Ecuador genera 540,7 millones de dólares (langostinos) y Chile 287,3 millones de dólares (salmón y trucha).

Una mención aparte merece China, que produce 13,38 millones de tm/año, con peces y plantas acuáticas cultivadas, siendo la producción de pescado cultivado el $8,7 \%$ del total de la acuicultura mundial. 


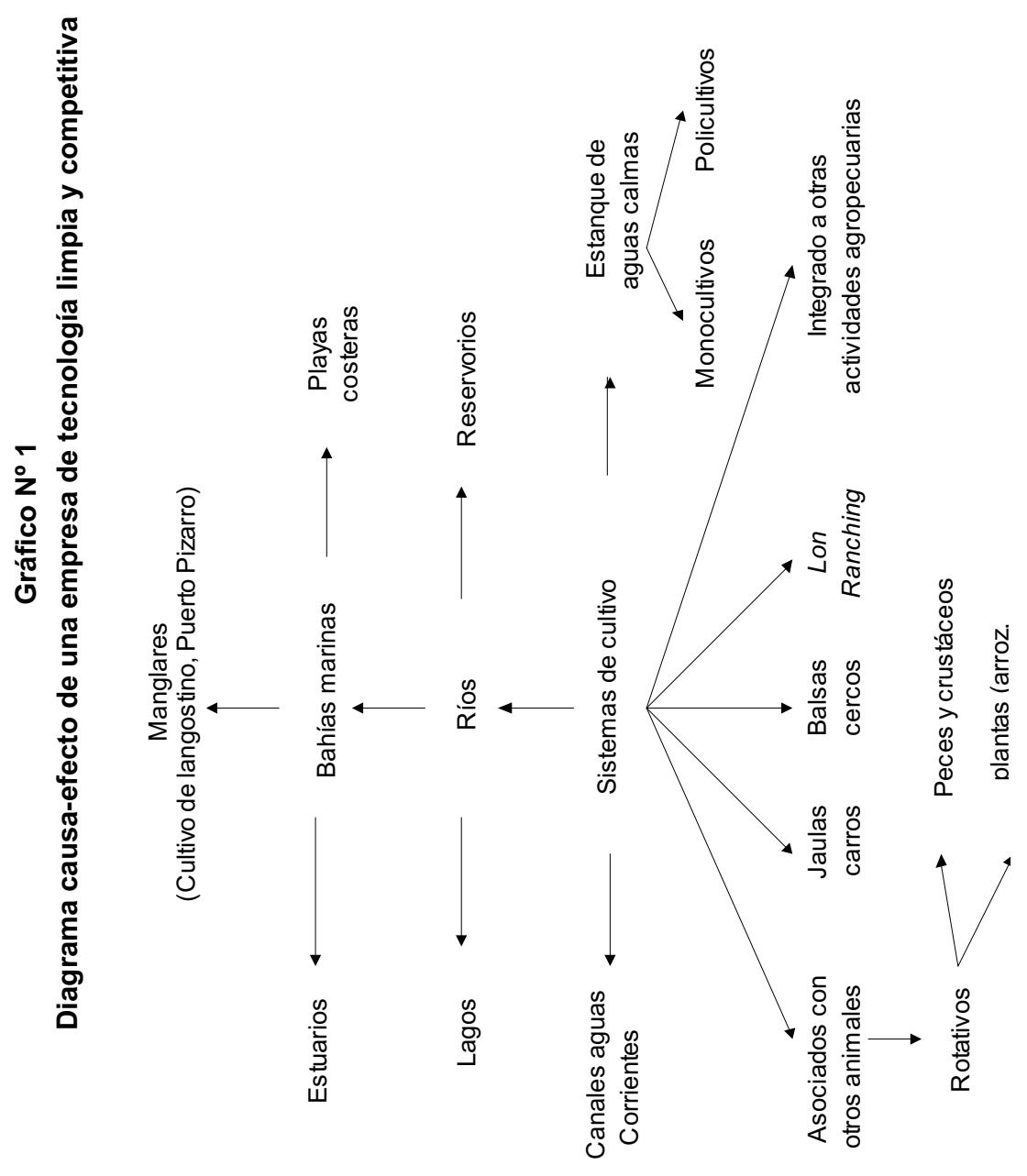

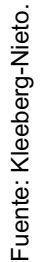




\section{Gráfico $N^{\circ} 2$}

Clasificación de recursos que pueden ser explotados en la acuicultura

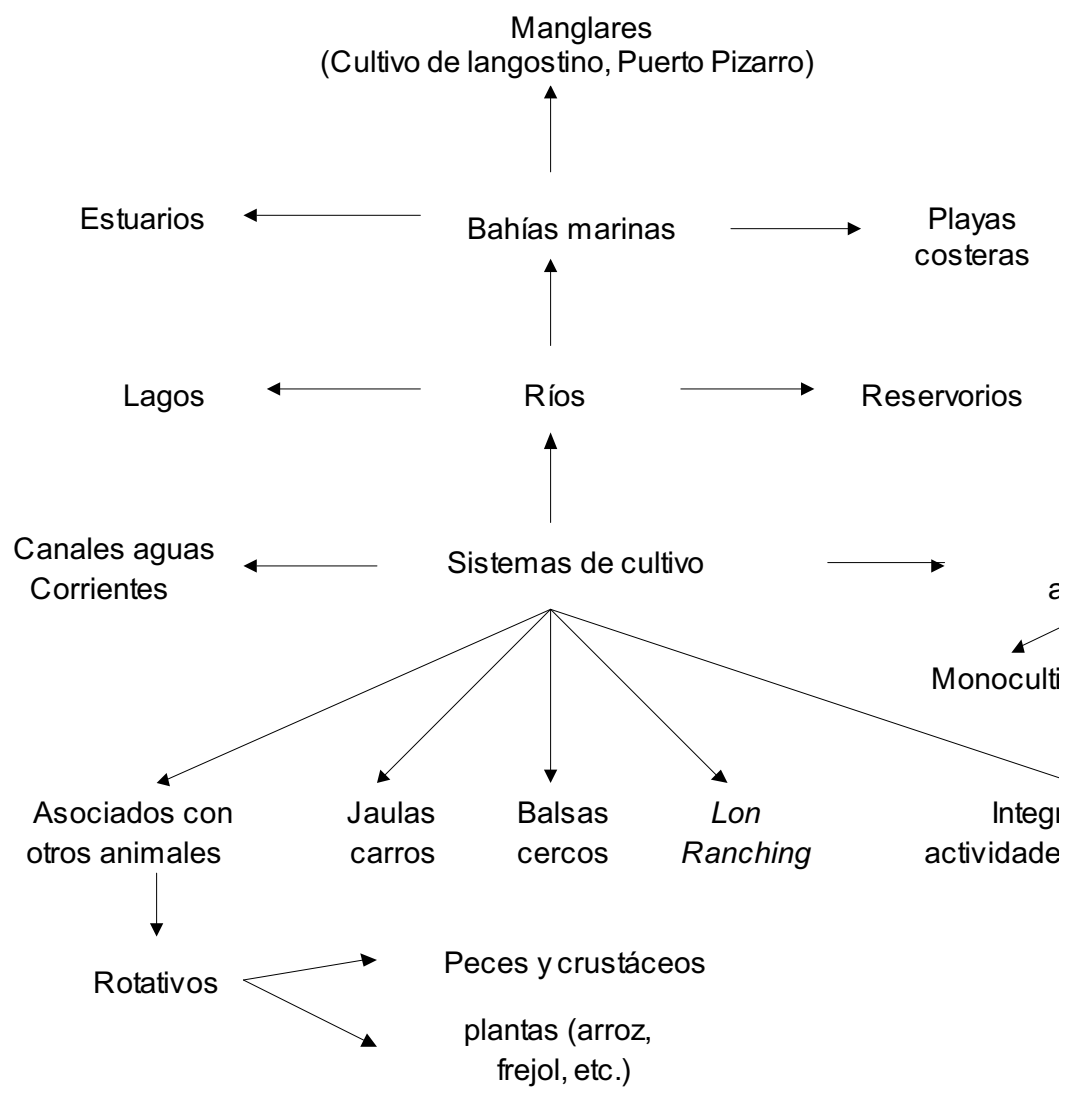




\section{Gráfico $\mathrm{N}^{\circ} 3$ \\ Lugares y sistemas de cultivo adecuado}

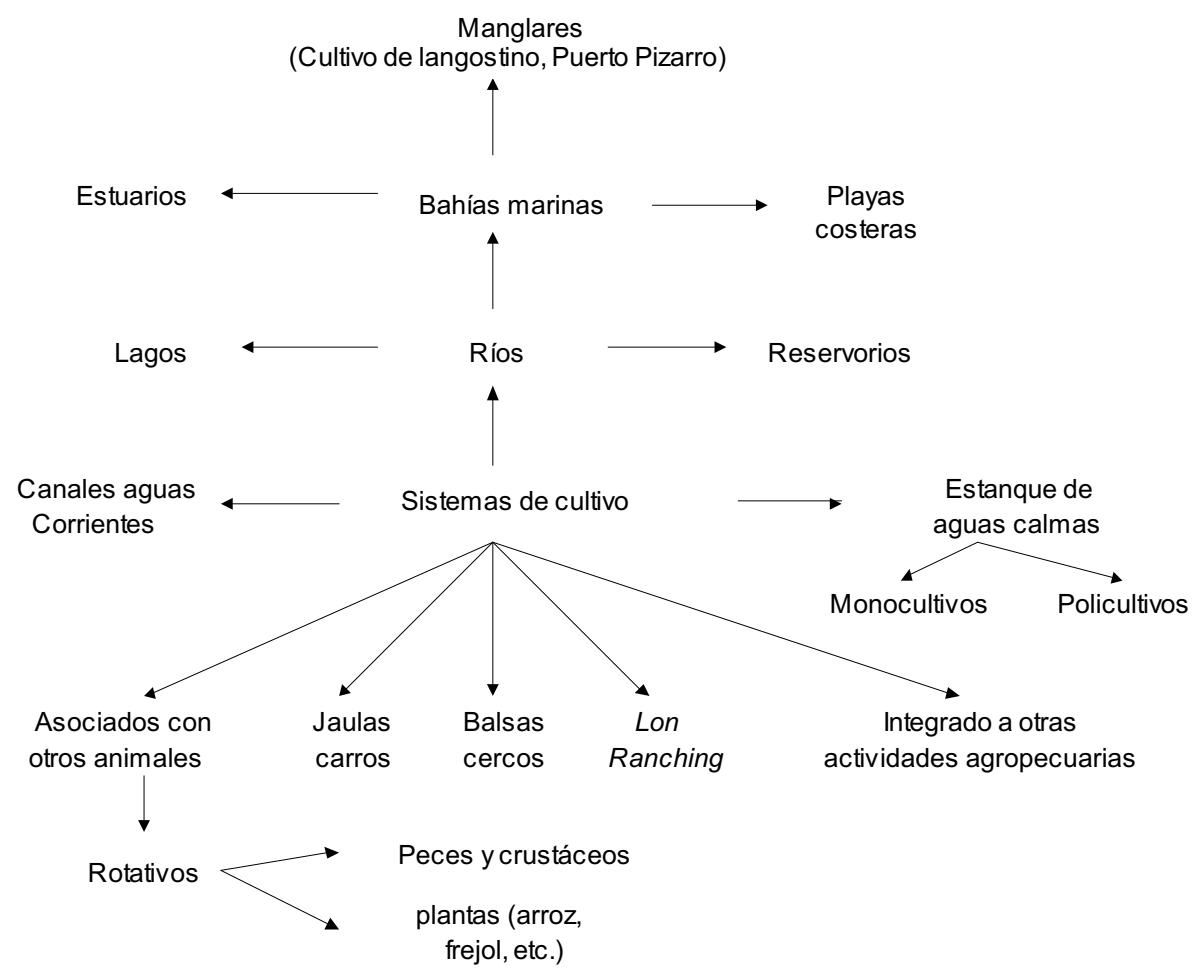

Fuente: Vinatea, 1999. 


\section{Bibliografía}

Arrús, J.A.

"Plan estratégico 1999-2004. Comisión de Plan Estratégico, Ministerio de Pesquería”. Ponencia presentada en la Universidad de Lima. Lima, setiembre de 1999.

Kleeberg, F. y M. Nieto

"Industria de las principales especies de importancia económica del sector pesquero: anchoveta, sardina, jurel, caballa y merluza". Lima: Universidad de Lima, 1997.

Vinatea, J.E.

"Perspectivas del desarrollo de la acuicultura en el Perú". Ponencia presentada en la Universidad de Lima. Lima, setiembre de 1999. 